\title{
Singular beams with transverse electric and transverse magnetic fields
}

\author{
T.A. Fadeyeva \\ V.I. Vernadsky Taurida National University, Simferopol, Ukraine, \\ E-mail: tatyana.fadeyeva@gmail.com
}

\begin{abstract}
We have obtained the solutions of the paraxial wave equation in free space and homogeneous media in the form of transverse electric (TE) and transverse magnetic (TM) fields with Laguerre-Gaussian (LG) and Hermite-Gaussian (HG) modulation functions. In contrast to the standard TE and TM beams we revealed that the axially symmetric field of LG mode beams have local elliptical polarization, while axial symmetry is totally broken down in the HG beams. The results obtained can be used for matching the fields inside the uniaxial crystal and free space.
\end{abstract}

Keywords: singular beam, TE mode, TM mode, polarization, optical vortex.

Manuscript received 01.11.12; revised version received 17.12.12; accepted for publication 26.01.13; published online 28.02.13.

\section{Introduction}

As a rule, the transverse electric (TE) and transverse magnetic (TM) mode beams are strongly associated with the azimuthally and radially polarized beams, respectively. They have unique properties: 1) their fields are linearly polarized in each point of the cross-section; 2) they are axially symmetric ones with a centered singular point where the directions of the linear polarizations are uncertain while their intensities vanish (see Fig. 1).

The physical aspect of such a unique wave construction is conditioned by two points: 1) the space variant linear polarization is shaped in such a way that the field projections onto the longitudinal $z$-direction vanish, 2) destructive interference obliterates any $z$ projections of the fields.

In the case of the azimuthally and radially polarized mode beams, it is the space variant polarization symmetry that is responsible for disappearance of the $z$ components, while the destructive interference does not play a part in the process. The physical reasons of it are related with the local structure of the medium where the beam field is transmitted. For example, the field TE and TM structures in the metal and dielectric circular waveguides are restricted by the demands of the boundary conditions. The birefringent medium of the uniaxial crystal admits the presence of the ordinary and extraordinary rays, when the electric and magnetic vectors must be parallel or perpendicular to the plane formed by the optical axis and propagation direction of the ray. Thus, the beams propagating along the crystal optical axis are of the TE and TM modes.

However, there is a great number of other TE and TM field modifications in natural where both the space variant of polarization and destructive interference take place in vanishing the $z$-components of the wave field at each point of the field cross-section that can be nonsymmetric with the space variant of elliptic polarization.

The aim of this paper is to consider the wave structure of TE and TM paraxial light beams. 


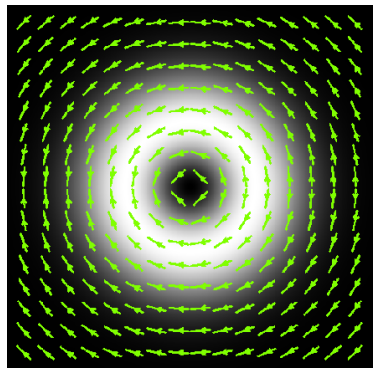

$T E, E_{z}=0$

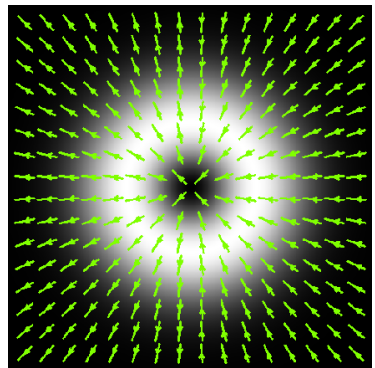

$T M, H_{z}=0$
Fig. 1. Azimuthally (TE) and radially (TM) polarized mode beams.

\section{Basic points}

It is well-known (see, e.g. [1]) that solutions of the Maxwell equations for monochromatic wave beams in a homogeneous medium can be reduced to the vector Helmholtz equation for the vector potential $\mathbf{A}$ under the condition of Lorentz gauge so that the electric $\mathbf{E}$ and magnetic $\mathbf{H}$ fields in free space are written as

$$
\mathbf{H}=\nabla \times \mathbf{A}, \quad \mathbf{E}=i k \mathbf{A}+i \nabla(\nabla \mathbf{A}) / k,
$$

where $k$ is a wavenumber. In the paraxial approximation $\left|\partial_{z}^{2} \mathbf{A}\right|<<\left|k^{2} \mathbf{A}\right|$, the transverse components can be written as

$\mathbf{H}_{\perp}=(\nabla \times \mathbf{A})_{\perp}, \mathbf{E}_{\perp}=i k \mathbf{A}_{\perp}$

and the additional condition

$$
\nabla \cdot(\varepsilon \mathbf{E})=0 \text {, }
$$

so that the longitudinal components of the electric and magnetic fields can be written out in terms of the transverse field components $\mathbf{E}_{\perp}, \mathbf{H}_{\perp}$ as

$$
E_{z} \approx i \nabla_{\perp} \mathbf{E}_{\perp} / k, H_{z} \approx i \nabla_{\perp} \mathbf{H}_{\perp} / k,
$$

where $\nabla_{\perp}=\mathbf{e}_{x} \partial_{x}+\mathbf{e}_{y} \partial_{y}$, while the complex amplitude $\tilde{\mathbf{A}}$ of the vector potential $\mathbf{A}=\tilde{\mathbf{A}}(x, y, z) \exp (i k z)$ obeys the vector paraxial equation

$$
\left(\nabla_{\perp}^{2}+2 i k \partial_{z}\right) \tilde{\mathbf{A}}_{\perp}=0 \text {. }
$$

It is convenient to describe the vector potential for the TE and TM vortex-beams in the basis of circular polarizations [2]: $A_{+}=A_{x}-i A_{y}, A_{-}=A_{x}+i A_{y}$ with the variables $\quad u=x+i y, \quad v=x-i y$, $\partial_{u} \equiv \partial_{x}-i \partial_{y}, \partial_{v} \equiv \partial_{x}+i \partial_{y}$.

In the case of the TE mode beam $\left(E_{z}=0, A_{z}=0\right)$ the equation (3) are fulfilled exactly so that:

$\partial_{v} A_{+}+\partial_{u} A_{-}=0$,

from whence

$A_{+}=\partial_{v} \Psi, \quad A_{-}=-\partial_{u} \Psi$,

while the generatrix function $\Psi$ is defined by the paraxial wave equation

$\left(\nabla_{\perp}^{2}+2 i k \partial_{z}\right) \Psi=0$.

Moreover, the demand (6) can be reinforced by the conditions
$\mathrm{TE}$

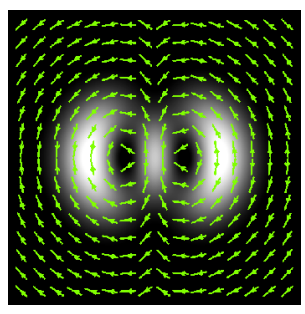

$\mathrm{TM}$

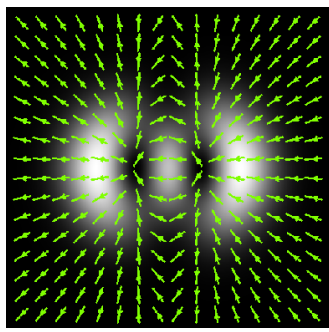

$$
m=1, n=0
$$
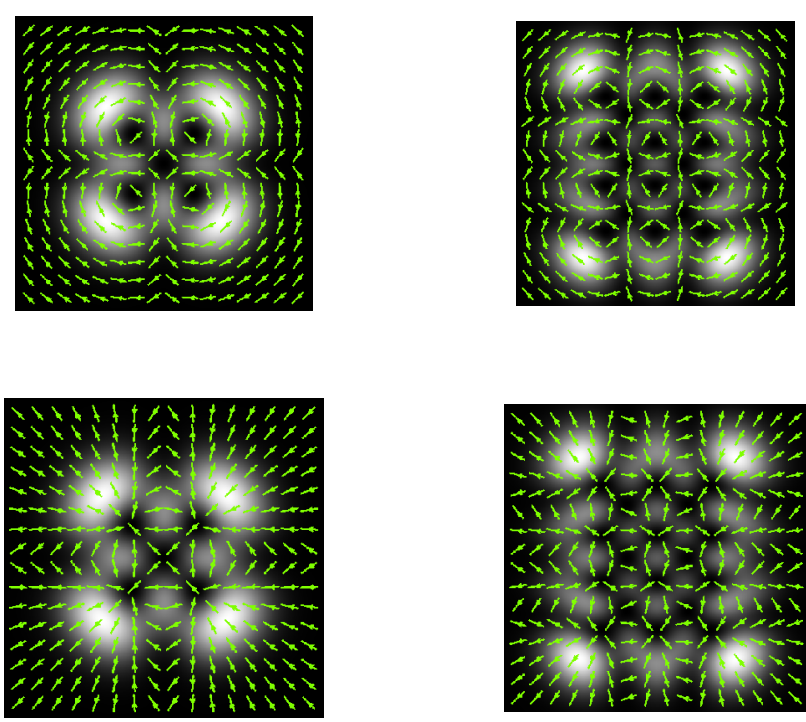

$m=1, n=1$

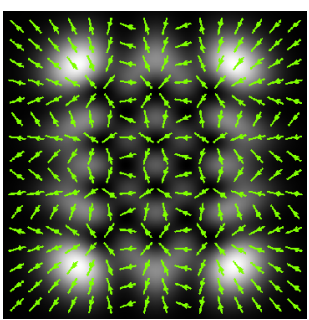

$m=3, n=2$

Fig. 2. TE and TM modes in the Hermite-Gaussian beams. 
$A_{+}=\partial_{v}^{n+1} \partial_{u}^{m} \Psi, \quad A_{-}=-\partial_{v}^{n} \partial_{u}^{m+1} \Psi$,

where $m, n=0,1,2, \ldots$ The vector-potential $\mathbf{A}$ obeys the wave equation (4) and the condition (2a).

Similar to that, the TM mode beams are defined by the conditions

$$
A_{+}=\partial_{v}^{n+1} \partial_{u}^{m} \Psi, \quad A_{-}=\partial_{v}^{n} \partial_{u}^{m+1} \Psi \text {. }
$$

\section{TE and TM modes in the standard paraxial beams}

\subsection{Hermite-Gaussian beams}

In the Cartesian frames and the linearly polarized basis, the condition (8) can be rewritten in the form

$$
A_{x}=\partial_{y}^{n+1} \partial_{x}^{m} \Psi, A_{y}=-\partial_{y}^{n} \partial_{x}^{m+1} \Psi
$$

for the TE beams and in the form

$$
A_{x}=\partial_{y}^{n} \partial_{x}^{m+1} \Psi, A_{y}=\partial_{y}^{n+1} \partial_{x}^{m} \Psi
$$

for the TM beams.

If the generatrix function $\Psi$ is chosen in the form of the Gaussian beam

$$
\Psi=\frac{1}{\sigma} \exp \left(-\frac{r^{2}}{w_{0}^{2} \sigma}\right)
$$

where $\sigma=1+i z / z_{0}, z_{0}=k w_{0}^{2} / 2, w_{0}$ stands for the radius of the beam waist, the conditions (2) and (10) give for the TE beams

$E_{x}=\frac{1}{\sigma^{(m+n+1) / 2}} H_{m}\left(\frac{x}{w_{0} \sqrt{\sigma}}\right) H_{n+1}\left(\frac{y}{w_{0} \sqrt{\sigma}}\right) \Psi$,
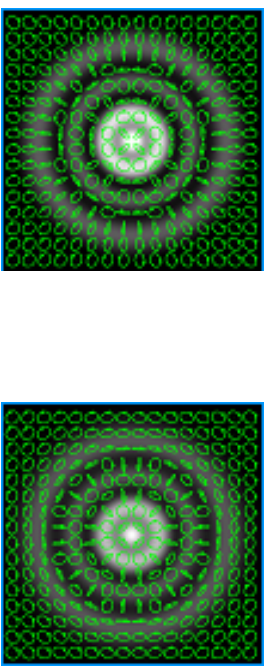

$$
m=1, n=3
$$

TE

TM

$$
E_{y}=-\frac{1}{\sigma^{(m+n+1) / 2}} H_{m+1}\left(\frac{x}{w_{0} \sqrt{\sigma}}\right) H_{n}\left(\frac{y}{w_{0} \sqrt{\sigma}}\right) \Psi,
$$

where $H_{m}(x)$ is the Hermite polynomials. For the TM beams, we obtain

$$
\begin{aligned}
& E_{x}=\frac{1}{\sigma^{(m+n+1) / 2}} H_{m+1}\left(\frac{x}{w_{0} \sqrt{\sigma}}\right) H_{n}\left(\frac{y}{w_{0} \sqrt{\sigma}}\right) \Psi, \\
& E_{y}=\frac{1}{\sigma^{(m+n+1) / 2}} H_{m}\left(\frac{x}{w_{0} \sqrt{\sigma}}\right) H_{n+1}\left(\frac{y}{w_{0} \sqrt{\sigma}}\right) \Psi,
\end{aligned}
$$

where we made use of the definition

$$
H_{m}(x)=(-1)^{m} e^{-x^{2}} \frac{d^{m}}{d x^{m}} e^{-x^{2}} \text {. }
$$

Fig. 2 illustrates the distributions of the electric field in the high order TE and TM modes beams on the background of the intensity distribution at the $z=0$ initial plane. The axial symmetry inherent to the lowest order mode beams gives place to the plane symmetry with the space variant of linear polarization. The azimuthally polarized areas occur only near the array of singular points with the zero intensity.

\subsection{Laguerre-Gaussian beams}

In the polar coordinates, the operators $\partial_{u}$ and $\partial_{v}$ take the form

$$
\partial_{u}=\frac{e^{-i \varphi}}{2}\left(\partial_{r}-\frac{i}{r} \partial_{\varphi}\right), \partial_{v}=\frac{e^{i \varphi}}{2}\left(\partial_{r}+\frac{i}{r} \partial_{\varphi}\right) .
$$

The equation (8) is reduced to the TE modes in the circularly polarized basis:
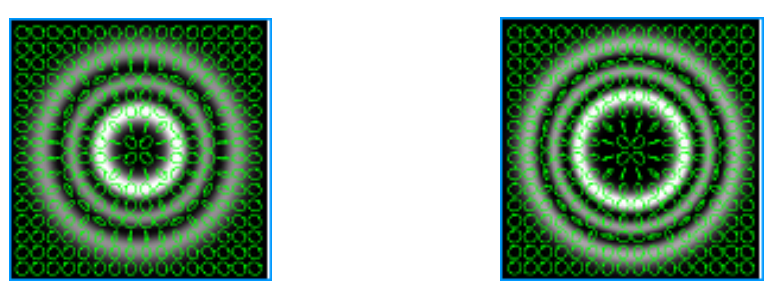

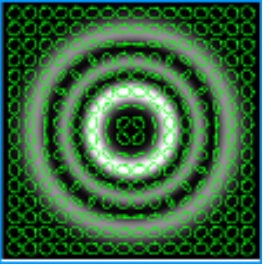

$m=4, n=3$

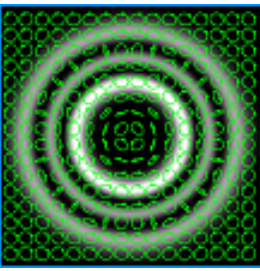

$m=10, n=3$

Fig. 3. TE and TM modes in the Laguerre-Gaussian beams. 


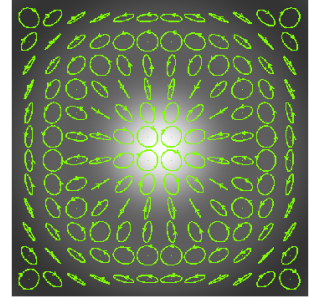

Fig. 4. Degenerated $C$-point.

$$
\begin{aligned}
& E_{+}=\left(\frac{r}{w_{0} \sigma}\right)^{|m-1|} e^{i(m-1) \varphi} L_{n}^{(m-1)}\left(\frac{r^{2}}{w_{0}^{2} \sigma}\right) \Psi, \\
& E_{-}=-\left(\frac{r}{w_{0} \sigma}\right)^{m+1} e^{i(m+1) \varphi} L_{n}^{(m+1)}\left(\frac{r^{2}}{w_{0}^{2} \sigma}\right) \Psi .
\end{aligned}
$$

For the TM mode we obtain

$$
\begin{aligned}
& E_{+}=\left(\frac{r}{w_{0} \sigma}\right)^{|m-1|} e^{i(m-1) \varphi} L_{n}^{(m-1)}\left(\frac{r^{2}}{w_{0}^{2} \sigma}\right) \Psi, \\
& E_{-}=\left(\frac{r}{w_{0} \sigma}\right)^{m+1} e^{i(m+1) \varphi} L_{n}^{(m+1)}\left(\frac{r^{2}}{w_{0}^{2} \sigma}\right) \Psi,
\end{aligned}
$$

where we made use of the definition of the associated Laguerre polynomials:

$$
L_{n}^{(m)}(x)=x^{m} e^{x} \frac{d^{n}}{d x^{n}}\left(x^{n+m} e^{x}\right) .
$$

Fig. 3 illustrates the structure of TE and TM modes in the Laguerre-Gaussian beams. Although the axial symmetry of the intensity and polarization distributions are conserved, there is light elliptically polarized in each space point. It comes to the front of the patterns of the TE and TM modes with $m=1$. There is not the intensity zero at the center. Instead of it, we observe the degenerated $C$-point shown in Fig. 4. The zero value of the $z$-components in the electric and magnetic fields at each point the beam cross-section is the result of the destructive interference.

\section{Conclusions}

The transverse electric TE and transverse magnetic TM mode beams have the azimuthally and radially polarized distributions only for the lowest order modes. In the rest cases, the axial symmetry is broken down although the transverse properties of the modes are conserved. The TE and TM modes of the higher orders in the HermiteGaussian beams have plane symmetry with the linear polarization over all the beam cross-section. Azimuthally and radially polarized distributions are observed only near singular points. The TE and TM modes in the Laguerre-Gaussian beams have the axially symmetric distributions of the intensity and polarization states. However, there is the space variant of elliptical polarizations.

\section{References}

1. S.R. Mishra, A vector wave analysis of a Bessel beam // Opt. Communs. 85, p. 159-161 (1991).

2. A. Volyar, V. Shvedov, T. Fadeyeva, Structure of nonparaxial Gaussian beams near the focus: 2 . Optical vortices // Optics and Spectroscopy, 90, p. 104-112 (2001).

3. T. Fadeyeva, C. Alexeyev, A. Rubass, A. Volyar, Vector erf-Gaussian beams: Fractional optical vortices and asymmetric TE and TM modes // Opt. Lett. 37(9), p. 1397-1399 (2012).

4. A.P. Kiselev, Localized light waves: Paraxial and exact solutions of the wave equation (a Review) // Optics and Spectroscopy, 102(4), p. 603-622 (2007).

5. I. Basisty, M. Soskin, M. Vasnetsov, Optical wavefront dislocations and their properties // Opt. Communs. 119, p. 604-612 (1995). 